Nervenarzt 2009 $\cdot 80: 5$

DOI 10.1007/s00115-008-2652-6

Online publiziert: 10. Januar 2009

๑) Springer Medizin Verlag 2009
W. Maier ${ }^{1} \cdot$ P. Falkai ${ }^{2}$

${ }^{1}$ Klinik und Poliklinik für Psychiatrie und Psychotherapie, Bonn

${ }^{2}$ Klinik für Psychiatrie und Psychotherapie, Universitätsmedizin Göttingen

\title{
Neues zur Diagnostik und Therapie schizophrener Psychosen
}

\section{Sehr geehrte Leserinnen, sehr geehrte Leser,}

liest man den Titel dieses Leitthemas „Neues zur Diagnostik und Therapie schizophrener Psychosen" möchte man sich gleich anderen Dingen zuwenden, in der Vorstellung, dieses Gebiet berge in sich doch keine wirklichen Innovationen. Wir hoffen aber sehr, dass es uns mit den vorliegenden Beiträgen gelungen ist, einige neue Aspekte aufzuzeigen, die auf dem Gebiet der schizophrenen Psychosen zurzeit intensiv bearbeitet werden.

Der Artikel von Mössner et al. sichtet den Stand molekulargenetischer Befunde sowohl im Bereich der Pathogeneseforschung als auch in der Pharmakogenetik. Die Autoren Gruber und Falkai diskutieren die neueste Datenlage zur Identifikation neuronaler Netzwerke als Korrelat kognitiver Störungen. Hier wird ausgehend von Arbeitsgedächtnisstörungen auf spezifische Störungsmuster hingewiesen, die in Zukunft sowohl differenzialdiagnostisch als auch differenzialtherapeutisch genutzt werden können.

Mittlerweile müsste bekannt sein, dass Psychotherapie und Schizophrenie keinen Widerspruch darstellen. Dementsprechend fasst der Beitrag von Klingberg und Buchkremer aktuelle Trends in der Psychotherapieforschung der Schizophrenie zusammen und weist auf zukünftige Entwicklungen hin. Einen weiteren Akzent setzt der Artikel von Becker und Mitarbeitern, der sich der Entwicklung von Versorgungsstrukturen für Patienten mit einer Schizophrenie widmet.
Zusammenfassend bietet Ihnen das in die vorliegende Ausgabe von „Der Nervenarzt“ integrierte Leitthema die Möglichkeit, sich in eine Reihe neuer Aspekte der Diagnostik und Therapie schizophrener Psychosen zu vertiefen. Wir hoffen, dass Ihnen das Studium der Beiträge Freude bereitet und Impulse für Ihre tägliche Arbeit entstehen.

Mit freundlichen Grüßen

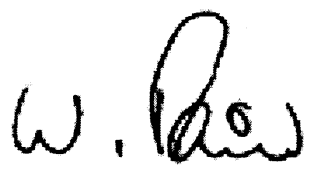

W. Maier, Bonn

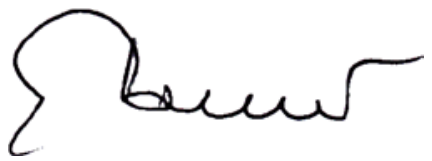

P. Falkai, Göttingen

\section{Korrespondenzadresse}

Prof. Dr. W. Maier

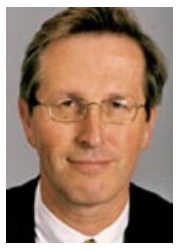

Klinik und Poliklinik für Psychiatrie und Psychotherapie Sigmund-Freud-Straße 25, 53105 Bonn Wolfgang.Maier@ ukb.uni-bonn.de

Prof. Dr. P. Falkai

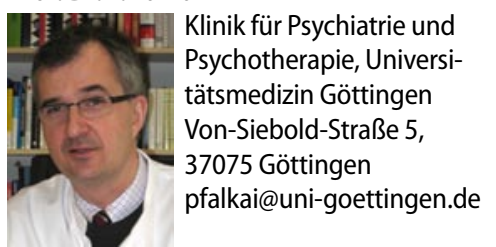

